\title{
Assessing the impact of discretisation assumptions in a length- structured population growth model
}

\author{
H. Drouineau ${ }^{a,{ }^{*}}$, S. Mahévas ${ }^{a}$, M. Bertignac ${ }^{b}$ and A. Fertin ${ }^{c}$ \\ a IFREMER Department EMH, Rue de I'lle d'Yeu, B.P. 21105, 44311 Nantes Cedex 3, France \\ ${ }^{\mathrm{b}}$ IFREMER Centre de Brest, Laboratoire de Biologie Halieutique, STH/LBH, BP 70, F-29280 Plouzané, France \\ c Université de Tours, IRBI UMR CNRS 6035, Parc Grandmont, 37200 Tours, France \\ *: Corresponding author : Drouineau H., email address : hdrouine@iffemer.fr
}

\begin{abstract}
:
Most of the traditional assessment models are age-structured. However, many biological and exploitation processes are more length-dependent than age-dependent, and the required length-age conversion of available data is often not reliable. Consequently, length-structured or age-length structured models have undergone considerable development in recent years. The growth transition matrix used to model the mean growth and growth variability of the population, is of primary importance in a length-structured matrix model. Building this growth transition matrix is not trivial and it is necessary to assess the impact that various assumptions may have to identify robust model structures. In this study, we assess the effects of (1) time and length discretisation, (2) the distribution of individuals within length classes and (3) the statistical distribution used to describe growth variability, by fitting a growth matrix model to individual quasi-continuous simulated growth data. The study quantitatively demonstrates that the choice of the time step and of length class width is the key point when building a length-structured population growth model. The use of a gamma distribution for the growth increments and/or a uniform distribution of individuals within length classes were found to make the model more robust.
\end{abstract}

Keywords: Length-structured model; Growth; Discrete model; Sensitivity analysis 
Introduction

19 From deterministic - single cohort analysis (Pope, 1972) to multi cohort analysis, age-structured

20 models (Fournier and Archibald, 1982 ; Virtala et al., 1998 ; Shepherd, 1999) are widely used for

21 fisheries stock assessments. While these models have proved to be useful in estimating population

22 abundance and assessing stocks, several limitations have been pointed out. One limit, often

23 mentioned, is related to the need to convert catch at size into catch at age, which is often carried out

24 using an age-length key. For some species, the difficulties in determining the age at length result in

25 uncertainty in catch-at-age. Moreover, since the ageing techniques are very expensive, few fishes

26 can be aged, further increasing the sampling error and variability in the age-length key. Another

27 source of uncertainty is the size-dependent selectivity function which can not be explicitly taken

28 into account in age-structured model though a age selectivity function may be used as a proxy.

29 Large interindividual and/or interannual variations in size within an age class could thus result in

30 incorrect estimates of fishing mortality by age. Consequently, length-structured models or age-

31 length structured models have undergone considerable development in recent years (Schnute, 1987 ;

32 Schnute et al., 1989a ; Schnute et al., 1989b ; Sullivan et al., 1990 ; Banks et al., 1991 ; De Leo and

33 Gatto, 1995 ; Fournier et al., 1998 ; DeLong et al., 2001 ; Froysa et al., 2002), but are still not often

34 used in routine stock assessments.

Different mathematical frameworks can be used to model length-structured population dynamics,

37 depending on whether population states are assumed discrete or continuous and whether the projection time is discrete or continuous. Continuous approaches better match biological processes.

39 However, the disadvantage of this approach is the complexity of handling differential equations

40 which do not necessarily have analytical solutions. In addition, available observed data are

41 generally aggregated thereby reducing the advantages of a continuous model. By contrast, discrete 
42 approximations of biological processes simplify model implementation and are easier to compute.

43 Discrete approaches require discrete assumptions on continuous processes. Following, discrete

44 growth modelling necessitates decisions on length classes width, time step duration, growth

45 increment model (stochastic or deterministic) and on the distribution of individuals within each

46 length class.

47 Fish growth is a major process of fish biology (Laslett et al., 2002) and is part of the information 48 necessary to estimate stock size and fishing mortality in stock assessments models (Anda-Montañez 49 et al., 1999). The importance of describing jointly mean growth and individual variability in growth 50 for stock assessment process has been widely acknowledged (Laslett et al., 2002 ; Chen et al., 51 2003). Two approaches are classically considered to model growth variability. The most common 52 model is a mean growth by length class given by a growth function, often a von Bertalanffy growth 53 curve, and a stochastic distribution for individual variability around the mean (Sullivan et al., 1990 ; 54 De Leo and Gatto, 1995 ; Cruywagen, 1997 ; Froysa et al., 2002). Another way to describe growth 55 variability is to integrate individual variability in the growth function with stochastic parameters 56 (Sainsbury, 1980 ; Smith et al., 1998 ; Smith and Botsford, 1998 ; Pilling et al., 2002). Several 57 approaches can be used to estimate growth parameters and corresponding variability. Length-at-age 58 can be back-calculated using information contained in fish hard parts, but this approach is often

59 imprecise due to difficulties in detecting growth increments. A second approach is tagging 60 (Shackell et al., 1997 ; Kendall and Nichols, 2002 ; Laslett et al., 2002 ; Eveson et al., 2004 ; de 61 Pontual et al., 2006). This technique is very expensive as many fishes have to be tagged in order to 62 be successful.

To date, very few studies have looked at the impact of time and length discretisation on the 65 ability of a population model to fit data and to estimate growth parameters. We chose in a first step 66 to work on a theoretical population only submitted to growth (no death and no recruitment) since it 
67 is a key process of length-structured model (Chen, 2003). In this study we provide a methodology

68 to assess the impact of several assumptions of time and length discretisation and growth increments

69 distribution in a length-structured population growth model. Data on individual growth trajectories

70 are rarely available. Thus, we produced several synthetic data sets of individual growth trajectories

71 corresponding to several hypotheses on growth increments. Then we fitted length-structured growth

72 population models to each synthetic data set. We provided from this analysis a flexible length-

73 structured population growth model.

\section{Data and methods}

\section{A length-structured population growth model}

A matrix formulation of a discrete time length-structured population growth model is presented

below. Since we only focus on the growth process, natural and fishing mortality are not described in the model. The length-structured abundance in number at time $t+1$ is given by the following

79 equation:

$$
N(t+1)=G \cdot N(t)
$$

where $N(t)$ : vector of abundance per size-class at time $t$ and $G$ is the matrix of probabilities of transition between length classes

We assumed a constant growth transition matrix over time (i.e., no seasonality has been taken

84 into account). The population is supposed to grow on average according to a von Bertalanffy

85 growth function, parameterised by three parameters $L_{\infty}, K$ and $T_{0}, \quad l(t)=L_{\infty} \times\left(1-\mathrm{e}^{-K\left(t-T_{0}\right)}\right) \cdot$ We

86 used an alternative formulation of this equation $l(t+\Delta t)-l(t)=\left(L_{\infty}-l(t)\right) \times\left(1-\mathrm{e}^{K \times \Delta t}\right)$

87 (Fabens, 1965) where $l(t+\Delta t)-l(t)$ is here the growth increments during a time step $\Delta t$ of an

88 individual of size $l(t)$ at the beginning of the time step. Growth increments within the population are 
random variables (Sullivan et al., 1990 ; Cruywagen, 1997 ; Froysa et al., 2002) whose mean is

90 given by the von Bertalanffy equation and whose variance is proportional to the square of its mean

91 (DeLong et al., 2001) (i.e., assuming a constant coefficient of variation of the growth increments):

92

$$
\left\{\begin{array}{c}
E(X / l)=\left(L_{\infty}-l\right) \cdot\left(1-\mathrm{e}^{K \cdot \Delta t}\right) \\
V(X / l)=C \cdot E^{2}(X / l)
\end{array}\right.
$$

with $X / l$ the random variable describing the growth increment for fish of initial size $l$ during a time step $\Delta t$ and $C$ a constant linking the mean to the variance equals to $C V^{2}(X / l)$.

Given the previous assumptions on the growth increments' mean and variance, the coefficients of the growth matrix were calculated according to the following equations:

- if fish size is assumed to be at the midpoint size of the departure class $i$, denoted $m_{\mathrm{i}}$, then the probability $g_{i j}$ of transition to class $j$ is given by the following single integral:

$$
g_{i j}=\int_{\left(m_{j}-\Delta l / 2\right)-m_{i}}^{\left(m_{j}+\Delta l / 2\right)-m_{i}} f(x) d x \text { with } \Delta l \text { the width of the length classes }
$$

- if fish size is assumed to be uniformly distributed within the departure class $i$, then the value of the following double integral is the probability $g_{i j}$ of transition from class $i$ to class $j:$

$$
\text { with } f \text { the assumed density function of growth increment. }
$$

We considered three different continuous distributions (normal, lognormal, gamma) to describe growth increments distribution (Table 1). The Gamma distribution (Table 2) is a two-parameters distribution, entirely defined by its two first moments. It is largely used in modelling thanks to its 
110 growth for small and large fish. The normal and lognormal (Table 2) statistical distributions were 111 also considered.

112 We considered classes of equal width in length and assumed two types of length classes (Table

113 1), which is the most common method to build classes (Sullivan et al., 1990 ; De Leo and Gatto,

1141995 ; Froysa et al., 2002).

115 Finally, three different time steps were considered in this analysis: monthly, quarterly and annual 116 (Table 1).

Let us denote $\operatorname{Mod}(\mathrm{Lm}, \Delta t, \Delta l, O D e p)$ the formulation of the length-structured population growth

119 model assuming a statistical distribution of growth increments $L m$, a time step $\Delta t$, length classes of

120 size $\Delta l$, and an option of distribution of individuals within classes ODep (Table 1). A simulation 121 corresponds to a ten-year cohort projection (we denoted $\mathrm{T}$ the number of time steps corresponding 122 to a ten-year projection) with an initial length distribution following a normal distribution of mean 5 $123 \mathrm{~cm}$ and standard deviation of 1.

\section{Synthetic Data}

125 Individual growth data are necessary to assess the impact of both time and length discretisation 126 assumptions in the growth dynamics model. However, no such data were available: consequently

127 we simulated individual growth trajectories. We used a monthly time step to mimic time continuous 128 growth. Shorter time steps were also tested, but the differences in frequencies-at-length were minor.

129 Let us denote $\boldsymbol{S}(\boldsymbol{K}, \boldsymbol{C} \boldsymbol{V}, \boldsymbol{L s})$ a simulation set of individual growth trajectories performed with, a 130 growth rate $\boldsymbol{K}$, a constant of variance of growth increments $\boldsymbol{C} \boldsymbol{V}$ and a statistical distribution of 131 growth increments $\boldsymbol{L s}$. A set of 10000 individual trajectories over a ten-year period was simulated 132 for each combination of assumptions of growth variability $(\boldsymbol{K}, \boldsymbol{C} \boldsymbol{V}, \boldsymbol{L s})$ using a monthly time step 
133 (Table 1). Two distinct von Bertalanffy growth rates $\boldsymbol{K}$ were considered (Table 1), respectively

134 corresponding to a slow growing species and a faster growing species. Three distinct constant of

135 variation were used (Table 1). These simulations aim at mimicking individual growth observations.

136 For each set, the initial length of each individual was generated according to a normal distribution

137 with mean $5 \mathrm{~cm}$ and standard deviation of 1.

1393 Analysis

$140 \quad 3.1$ Experimental design

141 We aimed at assessing the impact of discretisation assumptions on length-structured population

142 growth model ability to fit sets of individual growth trajectories and estimate growth parameters.

143 We used an experimental design (Kleijnen, 1998) that we analysed using standard linear models.

144 Each formulation of the length-structured population growth model $\operatorname{Mod}(\mathrm{Lm}, \Delta t, \Delta l$, Odep) was

145 fitted to each simulated data set of individual growth trajectories $S(\boldsymbol{K}, \boldsymbol{C V}, \mathbf{L s})$ by estimating Kest 146 and $C V$ which minimize the sum of squared error:

$$
L S(\text { Kest }, C V)=\sum_{i=1}^{I} \sum_{t=1}^{T}\left(f_{\text {est }}(t, i)-f_{\text {obs }}(t, i)\right)^{2}
$$

with $f o b s(t, i)$ the simulated frequencies of individuals within class $i$ at time step $t$, fest $(t, i)$ the

149 frequencies of individuals within class $i$ at time step $t$ estimated by the matrix model, $I$ the number

150 of classes and $T$ the number of time steps. This design required 648 simulations (18 sets of

151 individuals growth trajectories times 36 formulations of the length-structured population growth 152 model).

154 The minimisation of the function $\operatorname{LS}\left(K_{e s t}, C V\right)$ was carried out using a genetic algorithm coupled 155 with a quasi-Newton algorithm provided in the Autodif library (Otter Research Ltd. http://otter- 
rsch.com). The genetic algorithm was used in a first step to provide a starting point not too far from

157 the optimum for the Autodif algorithm.

158 For each experiment, the adequacy of the length-structured population growth model is 159 quantified using two criteria:

160 - The discrepancy between the $K$ estimated (Kest) by the matrix model and the $\boldsymbol{K}$ used to 161 generate the observations data $(\boldsymbol{K})$. It quantifies the goodness of the estimation of $K$

162 - The Bayesian Information Criterion $(B I C)$ is computed using the formula

$163 B I C=-2 \cdot \log L+p \cdot \log (n)$ with $p=2$ the number of estimated parameters $(K$ and $C V)$

$164 \log L=n \times \log (\sqrt{2 \times \pi})+\frac{1}{2} \times\left[n \times\left(\log \frac{n-1}{L S}-1\right)+1\right]$ and $\mathrm{n}$ the number of observations (number of 165 time steps $\mathrm{X}$ number of classes). The $B I C$ is here a measure of model ability to fit the data. The $B I C$ 166 balances the discrepancy between data and predictions (measured by LS) by the number of 167 estimated parameters and the number of observations (which depends on the time step and length 168 classes width in the model).

\subsection{Assessing discretisation assumptions}

We first used box-and-whisker plots of the estimated growth rate Kest for each modality of the

172 four assumptions in order to visually detect biases. Then, a statistical analysis of the criteria was

173 carried out to quantify the effect of each hypothesis. First, each criterion was analysed separately

174 using linear models step fitted on the transformed criterion, respectively $\log (|\mathrm{BIC}|)=L B i c$ (the

175 absolute value is necessary for the logarithmic transformation because the $B I C$ were negative) and

$176 \log (|\mathrm{K}-\mathrm{Kest}|)=L K$, where $L$ Bic and LK will refer to the two transformed variables. An appropriate

177 model is characterised by a high $L B i c$ and a low $\mid \boldsymbol{K}$-Kest $\mid$. A full experimental design allows 
178 inclusion of all the main effect and first-order interaction in the model (Kleijnen, 1998), so that the

179 models may be written under the general form:

$$
\begin{aligned}
& L B i c \sim \mu+L m+\Delta t+\Delta l+\text { Odep }+\boldsymbol{K}+\boldsymbol{C} \boldsymbol{V}+\boldsymbol{L s}+L m: \Delta t+L m: \Delta l+L m: \text { Odep }+L m: \boldsymbol{K}+ \\
& \quad L m: \boldsymbol{C} \boldsymbol{V}+L m: \boldsymbol{L s}+\Delta t: \Delta l+\Delta t: \text { Odep }+\Delta t: \boldsymbol{K}+\Delta t: \boldsymbol{C V}+\Delta t: \boldsymbol{L s}+\Delta l: \text { Odep }+\Delta l: \boldsymbol{K} \text { (1) }
\end{aligned}
$$$$
+\Delta l: \boldsymbol{C V}+\Delta l: \mathbf{L s}+\text { Odep }: \boldsymbol{K}+\text { Odep }: \boldsymbol{C} \boldsymbol{V}+\text { Odep }: \boldsymbol{L s}+\boldsymbol{K}: \boldsymbol{C V}+\boldsymbol{K}: \boldsymbol{L s}+\boldsymbol{C V}: \mathbf{L s}
$$

$$
L K \sim \mu+L m+\Delta t+\Delta l+\text { Odep }+\boldsymbol{K}+\boldsymbol{C} \boldsymbol{V}+\boldsymbol{L s}+L m: \Delta t+L m: \Delta l+L m: O d e p+L m: K+
$$

$L m: \boldsymbol{C V}+L m: \boldsymbol{L} \boldsymbol{s}+\Delta t: \Delta l+\Delta t:$ Odep $+\Delta t: \boldsymbol{K}+\Delta t: \boldsymbol{C} \boldsymbol{V}+\Delta t: \mathbf{L}+\Delta l:$ Odep $+\Delta l: \boldsymbol{K}$

$+\Delta l: \boldsymbol{C V}+\Delta l: \mathbf{L s}+$ Odep $: \boldsymbol{K}+$ Odep $: \boldsymbol{C V}+$ Odep $: \boldsymbol{L s}+\boldsymbol{K}: \boldsymbol{C V}+\boldsymbol{K}: \boldsymbol{L s}+\boldsymbol{C V}: \boldsymbol{L s}$

with $\alpha_{i}$ the effect of the assumption $i \beta_{i, j}$ the effect of the interaction between assumption $i$ and assumption $j$.

The coefficients of an effect are constrained to sum to 0 .

These linear models enable us to quantify the impact of the assumptions of both quality of $\mathrm{K}$ estimation and model fit. In addition, we were also interested in selecting the most appropriate model with respect to both objectives. A multiple regression tree (De'Ath, 2002) was built to explain the variations of the two transformed variables (previously normalized and centered) by the different explanatory variables (i.e., different assumptions). The model is (bold terms correspond to assumptions for the quasi-continuous model., other terms correspond to assumptions on the matrix model).:

$$
\frac{(\operatorname{Lbic}-\operatorname{mean}(\text { Lbic }))}{s d(\text { Lbic })}, \frac{(L K-\text { mean }(L K))}{s d(L K)} \sim \boldsymbol{\Delta t}+\boldsymbol{\Delta l}+\boldsymbol{L m}+\boldsymbol{C} \boldsymbol{V}+\boldsymbol{K}+\boldsymbol{O D e p}+\boldsymbol{L s}
$$

The tree is grown by repeated binary partitioning of the data, maximizing the Euclidean distance between the two group centroids and minimizes the total Euclidean distance to the centroid within each group. Each partition is characterized by an explanatory variable and the rank of the explanatory variable selections in the tree reflects the magnitude of impact of the variable on both 198 responses. 
200 Results

2011 Experimental designs analysis

202 Table 3 and Table 4 summarize the remaining effects of model (1) and (2) after performing 203 classical Fisher tests and residuals analysis. The $\mathrm{R}^{2}$ of the linear models are 0.995 for the $L B I C$, and 2040.674 for the $L K$. In those tables, the estimates quantify the strength of the effect of the considered 205 assumption. The reader should notice that for:

206 - model (1) the assumptions which have a positive estimate improve the fit to observed frequencies-at-length

- model (2) the assumptions which have a negative estimate improve the estimation of the growth rate.

211 Fitting observed frequencies-at-length (Table 3)

212 The significant effects of interaction factors are minor compared to the main effects and do not

213 influence the results. Among the main effects, most factors are significant, but few have a large

214 estimated value. Length class width and time step duration are the two factors which have the

215 strongest influence. The best fits were obtained with a monthly time step and $1 \mathrm{~cm}$ length classes

216 width. Results also demonstrated that a large inter-individual variability and a large growth rate are

217 more easily fitted by the matrix models.

2191.2 Ability to estimate the growth rate K (Table 4)

220 The box-and-whisker plots (Fig. 1) did not show any bias in the estimation of the growth rate, 
except when assuming a normal distribution for the growth increments. However, they showed that

222 the precision of the estimation varies across the different assumptions. The variability is particularly

223 high for a monthly time step, a normal distribution of growth increments and $5 \mathrm{~cm}$ classes.

224 The linear models showed that fewer factors have a statistically significant effect on the

225 estimation of the growth rate, however many more factors have a strong impact the estimation. As

226 previously, classes of $1 \mathrm{~cm}$ greatly improved K estimation. More surprisingly, short time steps do

227 not systematically improve the estimation of the growth rate. In fact, with a monthly (or quarterly)

228 step, individuals have a too small probability to change class (especially when classes are $5 \mathrm{~cm}$

229 wide) so that $\mathrm{K}$ tends to be over-estimated to compensate. This highlights the links existing between

230 size classes and time steps (the interaction of the two factors is strongly significant). The choice of a

231 time step should be strongly linked to the choice of the size of classes, both choices being also

232 constrained by the available data.

233 The linear model demonstrates that, in contrast with the BIC analysis, a low growth rate and a

234 weak inter-individual variability favour a good estimation of K. Furthermore, if the individuals are

235 uniformly distributed within classes, the estimation is improved, especially with a short time step.

236 Finally, it highlights the fact that gamma and lognormal distributions used in the length-

237 structured population growth model yield very similar results. The gamma distribution tends

238 however to produce better fits than the lognormal even if a lognormal distribution is assumed for $S$.

239 A normal assumption in the length-structured population growth model gives poor estimation of $\boldsymbol{K}$

240 when a gamma or lognormal distribution is assumed for $S$. The gamma distribution for Mod give

241 better results than a normal distribution even when a normal distribution is assumed for $S$ (this can

242 be checked by summing the estimates of the main effects and of the interaction). Among the three

243 distributions tested, the gamma distribution appears to be the most flexible distribution to represent

244 the variability of the growth increments. 
247 The first criterion to partition groups of fits is the width of the classes. Small length classes tend

248 to improve the estimation of $\mathrm{K}$ and especially the goodness of the fit of frequencies at length. Then

249 the fits are partitioned according to time steps : large time steps degrade the estimation of

250 frequencies at length, and this is amplified with wide length classes. We can notice that monthly

251 and quarterly time steps are opposed to yearly time step The next two partitions concern the

252 assumption on statistical distribution of growth increments: assuming a gamma or lognormal

253 improves the goodness of the fit.

\section{Discussion}

256 This study aimed at analysing the impact of various assumptions on a length-structured

257 population growth model, especially time and length discretisation, in order to detect some artefacts

258 caused by inappropriate assumptions in growth model and to provide the most flexible model. This

259 kind of sensitivity analysis is essential in a discrete model and the methods we provide can be

260 applied in many situations. This study is a preliminary analysis to the development of a complete

261 length-structured model for European hake. In this paper, we chose to only focus on growth because

262 it is a critical point in length-structured model of population dynamics. A von Bertalanffy growth

263 curve was used to model mean growth, but more general growth functions can be considered as

264 polynomial, Gompertz, Verhulst, Richards, Schnute. Inter-individual variability was modelled using

265 a statistical distribution around this mean. Theoretically, a growth increment variance could be

266 estimated for each length class but we made this assumption to reduce the number of parameters to

267 estimate. It should be noticed that this relationship between the mean and variance constrains the

268 statistical distributions. A similar analysis has been carried out assuming a growth increment

269 variance proportional to the mean (Sullivan et al., 1990 ; Sullivan, 1992) (not presented here)

270 leading to very similar results. The Beta-Binomial distribution (Ennis and Bi, 1998) was primarily 
used to model the distribution of growth increments, but rejected because of unsatisfactory results.

272

273

274

275

276

277

278

279

280

281

282

Individual growth data are necessary to assess the impact of both time and length discretisation assumptions in the length-structured population growth model. Tagging data would have been an interesting data source, but despite a recent tagging survey, the amount of tagging data for European hake, the species we are currently working on, was insufficient. Consequently we decided to simulate individual growth data, trying to mimic an average von Bertalanffy growth and introducing inter-individual variability. It may have been interesting to simulate individual growth trajectories considering a population with stochastic $\mathrm{K}$ and $\mathrm{L}_{\infty}$. The results may largely be influenced by the methods used to generate these individual growth trajectories and therefore should not be generalized in the broader context. More generally, the results we obtain are not generic since they depend (1) on the method used to simulate data (2) on the considered model, species and/or fisheries. However, the methodology we provide is generic and is appropriate to assess the impact of any discretisation processes in a discret model.

In this analysis, we considered $\mathrm{L}_{\infty}$ was known because it may be approximated by a function of Lmax and it avoids the problem of the correlation between $\mathrm{K}$ and $\mathrm{L}_{\infty}$. This assumption has already been used for example in DeLong et al. (2001). The sets of von Bertalanffy parameters used in the study are closed to those currently used for stock assessment of European hake $(\mathrm{K}=0.1)$ (Piñeiro and Sainza, 2003) and to the recently assumed growth $(\mathrm{K}=0.2)$ (de Pontual et al., 2003 ; Kacher and Amara, 2005 ; de Pontual et al., 2006). The growth rate $K$ and the coefficient of variation $C V$ were estimated by minimising the sum of squared deviations between frequencies estimated by the length-structured population growth model and simulated frequencies-at-length.

We chose two criteria to describe the goodness of fit of the length-structured population growth model to frequencies generated by the quasi-continuous model. Those two criteria correspond to two modelling aims. The first objective was to reproduce and/or predict the observed data. In that case, we are less interested in the biological processes under-lying the model than in its ability to 
296

297

298

299

300

301

302

303

304

305

reproduce observations. The other objective was to reproduce the biological process driving the model (for example growth). In that case, the quality of the estimates (precision, bias) is much more important. Selecting a model that achieves these two objectives should be of major importance. However, it requires a method combining these two criteria. Indeed, in this study we showed that the best fits (the ones with the lowest $B I C$ ) are not necessarily the ones which provide the best estimates of unknown parameters. Until now, we have not found any satisfactory quantitative methods to simultaneously assess the effect of the different assumptions on both criteria. A possible solution would be to build a desirability function (Harrington, 1965) combining criteria, but the choice of the function is rather subjective since the user must first define individual desirability functions for each response. Multivariate regression trees provide a convenient graphical tool to investigate the impact of assumptions in the length-structured population growth model on both criteria simultaneously but this is only an explanatory analysis based on hierarchical clustering.

$$
\text { The statistical analysis of the two criteria enables identification of robust model structures for the }
$$
length-structured population growth model. It demonstrated that the choice of the time step and of length class width was the key point when building a length-structured population growth model. These choices are closely linked to available data and are strongly interdependent. A compromise needs to be found to comply with the two modelling objectives of the model. Other assumptions had a significant effect on the estimation of the growth rate. The gamma distribution was the most flexible statistical distribution to describe growth increments variability. Assuming that individuals are uniformly distributed within length classes, rather than centred, also improved significantly the estimation of the growth rate. We only tried two kinds of distribution of individuals within length classes though some other distributions are sometimes used, for example U-shaped distribution which is especially appropriate for wide length classes. It would also be interesting to assess the impact of the number of individual growth trajectories to quantify the number of trajectories required for a reliable growth rate estimation. 
321 This study was a preliminary analysis in the development of a length-based population model for 322 the European hake (Merluccius merluccius) stock dynamics. The results inform on the selection of 323 appropriate assumptions of time and length discretisation for this model. The European hake

324 dynamic model will have a quarterly time step and $1 \mathrm{~cm}$ width classes since these appear to be a 325 relevant compromise and they correspond to the time-scale of available data. A gamma distribution 326 will be used to describe the variability of growth increments and individuals will be uniformly 327 distributed within length classes.

\section{Acknowledgements}

We thank Mike Smith, José De Oliveira and Dominique Pelletier for their suggestions and criticisms which largely enhanced clarity and precision in this paper.

\section{References}

331 Anda-Montañez, A. D., Arreguin-Sanchez, F., and Martinez-Aguilar, S. 1999. Length-based growth 332 estimates for Pacific sardine (Sardinops sagax) in the Gulf of California, Mexico. CalCOFI 333 Reports, 40: 179-183.

334 Banks, H. T., Botsford, L. W., Kappel, F., and Wang, C. 1991. Estimation of growth and survival in 335 size-structured cohort data: an application to larval striped bass (Morone saxatilis). Journal of 336 Mathematical Biology, 30: 125-150.

337 Chen, Y., Hunter, M., Vadas, R., and Beal, B. 2003. Developing a growth-transition matrix for the 338 stock assessment of the green sea urchin (Strongylocentrotus droebachiensis) off Maine. Fishery 339 Bulletin, 101: 737-744. 
340 Cruywagen, G. 1997. The use of generalised linear modelling to determine inter-annual and inter-

341 area variation of growth rates : the caperock lobster as example. Fisheries Research, 29: 119-131.

342 De Leo, G. A., and Gatto, M. 1995. A size and age-structured model of the European eel (Anguilla 343 anguilla L.). Canadian Journal of Fisheries and Aquatic Sciences, 52: 1351-1367.

344 de Pontual, H., Bertignac, M., Battaglia, A., Bavouzet, G., Moguedet, P., and Groison, A. L. 2003. 345 A pilot tagging experiment on European hake (Merluccius merluccius): methodology and 346 preliminary results. ICES Journal of Marine Science, 60: 1318-1327.

347 de Pontual, H., Groison, A. L., Pineiro, C., and Bertignac, M. 2006. Evidence of underestimation of 348 European hake growth in the Bay of Biscay, and its relationship with bias in the agreed method 349 of age estimation. ICES Journal of Marine Science, 63: 1674-1681.

350 De'Ath, G. 2002. Multivariate Regression Trees: a new technique for modelling species351 environment relationships. Ecology, 83: 1105-1117.

352 DeLong, A. K., Collie, J. S., Meise, C. J., and Powell, J. C. 2001. Estimating growth and mortality 353 of juvenile winter flounder, Pseudopleuronectes americanus, with a length-based model. 354 Canadian Journal of Fisheries and Aquatic Sciences, 58: 2233-2246.

355 Ennis, D. M., and Bi, J. 1998. The beta-binomial model: Accounting for intertrial variation in 356 replicated difference and preference tests. Journal of Sensory Analysis, 13: 348-412.

357 Eveson, J. P., Laslett, G. M., and Polacheck, T. 2004. An integrated model for growth incorporating 358 tag-recapture, length frequency, and direct aging data. Canadian Journal of Fisheries and Aquatic $359 \quad$ Sciences, 61: 292-306.

360 Fabens, A. J. 1965. Properties and fitting of the von Bertalanffy growth curve. Growth, 29: 
362 Fournier, D. A., Hampton, J., and Sibert, J. R. 1998. MULTIFAN-CL: a length-based, age363 structured model for fisheries stock-assessment, with application to South Pacific albacore, 364 Thunnus alalunga. Canadian Journal of Fisheries and Aquatic Sciences, 55: 2105-2116.

365 Fournier, D., and Archibald, C. P. 1982. A general theory for analysing catch-at-age data. Canadian 366 Journal of Fisheries and Aquatic Sciences, 39: 1195-1207.

367 Froysa, K. G., Bogstad, B., and Skagen, D. W. 2002. Fleksibest- an age-length structured fish stock 368 assessment model. Fisheries Research, 55: 87-101.

369 Harrington, E. C. J. 1965. The desirability function. Industrial Quality Control, 21: 494-498.

370 Kacher, M., and Amara, R. 2005. Distribution and growth of 0-group European hake in the Bay of 371 Biscay and Celtic Sea: a spatial and inter-annual analyses. Fisheries Research, 71: 373-378.

372 Kendall, W. L., and Nichols, J. D. 2002. Estimating state-transition probabilities for unobservable 373 states using capture-recapture/resighting data. Ecology, 83: 3276-3284.

374 Kleijnen, J. P. C. 1998. Experimental design for sensitivity analysis, optimization, and validation of 375 simulations model. In Handbook of simulation :principles, methodology, advances, applications, 376 and practice. Banks, J. (Ed.), New York. 173-224.

377 Laslett, G. M., Eveson, J. P., and Polacheck, T. 2002. A flexible maximum likelihood approach for 378 fitting growth curves to tag-recapture data. Canadian Journal of Fisheries and Aquatic Sciences, $379 \quad$ 59: $976-986$.

380 Pilling, G. M., Kirkwood, G. P., and Walker, S. G. 2002. An improved method for estimating 381 individual growth variability in fish, and the correlation between von Bertalanffy growth 
383 Piñeiro, C., and Sainza, M. 2003. Age estimation, growth and maturity of the European hake 384 (Merluccius merluccius (Linnaeus, 1758)) from Iberian Atlantic waters. ICES Journal of Marine 385 Science, 60: 1086-1102.

386 Pope, J. G. 1972. An investigation of the accuracy of virtual population analysis for the calculation 387 of fishing mortality from catch at age data. International Commission for the Northwest Atlantic $388 \quad$ Fisheries Research Bulletin, 9: 65-74.

389 Sainsbury, K. J. 1980. Effect of Individual Variability on the von Bertallanffy Growth Equation. 390 Canadian Journal of Fisheries and Aquatic Sciences, 377: 241-247.

391 Schnute, J. 1987. A general fishery model for a size-structured fish population. Canadian Journal of $392 \quad$ Fisheries and Aquatic Sciences, 44: 924-940.

393 Schnute, J. T., Richards, L. J., and Cass, A. J. 1989a. Fish survival and recruitment: investigations 394 based on a size-structured model. Canadian Journal of Fisheries and Aquatic Sciences, 46: $395 \quad 743-769$.

396 Schnute, J. T., Richards, L. J., and Cass, A. J. 1989b. Fish growth: investigations based on a size397 structured model. Canadian Journal of Fisheries and Aquatic Sciences, 46: 730-742.

398 Shackell, N. L., Stobo, W. T., Franck, K. T., and Brickman, D. 1997. Growth of cod (Gadus 399 morhua) estimated from mark-recapture programs of the Scotian Shelf and adjacent areas. ICES $400 \quad$ Journal of Marine Science, 54: 383-398.

401 Shepherd, J. G. 1999. Extended survivors analysis: an improved method for the analysis of catch-at402 age data and abundance indices. ICES Journal of Marine Science, 56: 584-591. 
403 Smith, B. D., and Botsford, L. W. 1998. Interpretation of growth, mortality, and recruitment 404 patterns in size-at-age, growth increment, and size frequency data. Proceedings of the North 405 Pacific Symposium on Invertebrate Stock Assessment and Management in Can. J. Fish. Aquat. 406 Sci., 125: 125-139.

407 Smith, B. D., Botsford, L. W., and Wing, S. R. 1998. Estimation of growth and mortality 408 parameters from size frequency distributions lacking age patterns: the red sea urchin 409 (Strongylocentrotus franciscanus) as an example. Canadian Journal of Fisheries and Aquatic $410 \quad$ Sciences, 55: 1236-1247.

411 Sullivan, P. J. 1992. A Kalman Filter Approach to Catch-at-Length Analysis. Biometrics, 48: $412 \quad 237-257$.

413 Sullivan, P. J., Lai, H., and Galluci, V. F. 1990. A catch -at- length analysis that incorporates a 414 stochastic model of growth. Canadian Journal of Fisheries and Aquatic Sciences, 47: 184-198.

415 Virtala, M., Kuikka, S., and Arjas, E. 1998. Stochastic virtual population analysis. ICES Journal of 416 Marine Science, 55: 892-904. 
419 Table 1: List of the abbreviations and their specifications. Bold terms correspond to assumptions

420 on the quasi-continuous model. Other terms correspond to assumptions on the length-structured 421 population growth model.

\begin{tabular}{|c|c|c|}
\hline Abbreviation & Signification & Possible Values \\
\hline$\Delta t$ & $\begin{array}{l}\text { Time steps of the length-structured population growth } \\
\text { model }\end{array}$ & $\begin{array}{l}\text { 12: monthly time step } \\
\text { 4: quaterly } \\
\text { 1: yearly }\end{array}$ \\
\hline$\Delta l$ & $\begin{array}{l}\text { Size of the length classes in the length-structured population } \\
\text { growth model }\end{array}$ & $\begin{array}{l}1: 1 \mathrm{~cm} \\
5: 5 \mathrm{~cm}\end{array}$ \\
\hline $\mathrm{Lm}$ & $\begin{array}{l}\text { Statistical distribution of the growth increments in the } \\
\text { length-structured population growth model }\end{array}$ & $\begin{array}{l}\text { 0: gamma } \\
\text { 1: normal } \\
\text { 2: lognormal }\end{array}$ \\
\hline ODep & $\begin{array}{l}\text { Distribution of the individual within length classes in the } \\
\text { length-structured population growth model }\end{array}$ & $\begin{array}{l}0: \text { center } \\
1: \text { uniform }\end{array}$ \\
\hline$C-C V$ & $\begin{array}{l}\text { Constant of variance in the quasi-continuous model - } \\
\text { corresponding coefficient of variance }\end{array}$ & $\begin{array}{l}0.4-0.6 \\
1-1 \\
4-2\end{array}$ \\
\hline $\boldsymbol{K}$ & Growth rate in the quasi-continuous model & $0.1,0.2$ \\
\hline$L s$ & $\begin{array}{l}\text { Statistical distribution of the growth increments in the } \\
\text { quasi-continuous model }\end{array}$ & $\begin{array}{l}0: \text { gamma } \\
\text { 1: normal } \\
\text { 2: lognormal }\end{array}$ \\
\hline$\alpha$ & Parameter of the Beta-Binomial & $0.1,1,10,50$ \\
\hline$\beta$ & Parameter of the Beta-Binomial & $1,10,50,100$ \\
\hline Kest & $\begin{array}{l}\text { Estimated growth rate when fitting the length-structured } \\
\text { population growth model on frequencies at length generated } \\
\text { by the quasi-continuous model }\end{array}$ & \\
\hline$B I C$ & $\begin{array}{l}\text { Bayesian Information Criterion when fitting the length- } \\
\text { structured population growth model on frequencies at length } \\
\text { generated by the quasi-continuous model }\end{array}$ & \\
\hline Lbic & Neperian logarithm of the absolute value of $B I C$ & \\
\hline$L K$ & $\begin{array}{l}\text { Neperian logarithm of the absolute value of the difference } \\
\text { bewteen Kest and } \boldsymbol{K}\end{array}$ & \\
\hline
\end{tabular}


423 Table 2: Distribution, mean and variance of the normal (N), lognormal (LN) and Gamma (G)

424 statistical distributions. With $\Gamma(x)=\int_{0}^{\infty} \mathrm{e}^{u} u^{x-1} d u$ the gamma function.

\begin{tabular}{cccc}
\hline Distrib & Density & $\mathrm{E}$ & $\mathrm{V}$ \\
\hline $\mathrm{N}$ & $f(x)=\frac{1}{\sigma \times \sqrt{(2 \times \pi)}} \times \mathrm{e}^{-\frac{1}{2} \times\left(\frac{x-\mu}{\sigma}\right)^{2}}$ & $E(X)=\mu$ & $V(X)=\sigma^{2}$ \\
$\mathrm{LN}$ & $f(x)=\frac{1}{\sigma \times x \times \sqrt{(2 \times \pi)}} \times \mathrm{e}^{-\frac{1}{2} \times\left(\frac{\log (x)-\mu}{\sigma}\right)^{2}}$ & $E(X)=\mathrm{e}^{\mu+\frac{\sigma^{2}}{2}}$ & $V(X)=\left(\mathrm{e}^{\sigma^{2}}-1\right) \times \mathrm{e}^{2 \mu+\sigma^{2}}$ \\
$\mathrm{G}$ & $f(x)=\frac{1}{\beta^{\alpha}} x^{\alpha-1} e^{-\frac{x}{\beta}}$ & $E(X)=\frac{\alpha}{\beta}$ & $V(X)=\frac{\alpha}{\beta^{2}}$ \\
\hline
\end{tabular}

425

426 
427 Table 3: Significant effects in the linear model explaining LBic of the fits by the different 428 assumptions .

$429 \quad * * *($ respectively $* *)$ stands for a p-value $<0.01$ (respectively $<0.05)$

\begin{tabular}{|c|c|c|}
\hline & Estimate & P-value \\
\hline Intercept & 8.92 & $* * *$ \\
\hline$L m=0$ & 0.03 & $* * *$ \\
\hline$L m=1$ & -0.05 & $* * *$ \\
\hline$\Delta t=1$ & -1.18 & $* * *$ \\
\hline$\Delta t=4$ & 0.06 & $* *$ \\
\hline$\Delta l=1$ & 1.37 & $* * *$ \\
\hline$C V=0.2$ & -0.21 & $* * *$ \\
\hline$K$ & 0.89 & $* * *$ \\
\hline$L s=0$ & 0.05 & $* *$ \\
\hline$L s=1$ & -0.06 & $* * *$ \\
\hline$O D e p=0$ & -0.03 & $* * *$ \\
\hline$\Delta t=1: \Delta l=1$ & -0.07 & $* * *$ \\
\hline$\Delta t=4: \Delta l=1$ & 0.04 & $* * *$ \\
\hline$\Delta t=1: K$ & 0.67 & $* * *$ \\
\hline$\Delta t=1: L m=0$ & -0.02 & $* *$ \\
\hline$\Delta t=1: L m=1$ & 0.03 & $* * *$ \\
\hline$\Delta t=4: L m=1$ & 0.02 & $* *$ \\
\hline$\Delta t=1: L s=1$ & 0.04 & $* * *$ \\
\hline$\Delta l=1: K$ & -0.49 & $* * *$ \\
\hline$\Delta l=1: L s=0$ & -0.04 & $* * *$ \\
\hline$\Delta l=1: L s=1$ & 0.06 & $* * *$ \\
\hline$L m=1: C V=0.2$ & 0.03 & $* * *$ \\
\hline$C V=0.2: K$ & 0.31 & $* *$ \\
\hline$C V=1: K$ & 0.22 & $* *$ \\
\hline$C V=0.2: L s=0$ & -0.05 & $* * *$ \\
\hline$C V=0.2: L s=1$ & 0.08 & $* * *$ \\
\hline$K: L s=1$ & -0.42 & $* * *$ \\
\hline
\end{tabular}

430 
431 Table 4: Significant effects in the linear model explaining $L K$ of the fits by the different 432 assumptions.

$433 \quad * * *($ respectively $* *)$ stands for a p-value $<0.01$ (respectively $<0.05)$

\begin{tabular}{lll}
\hline & Estimate & P-value \\
\hline Intercept & -4.74 & $* * *$ \\
$\Delta t=1$ & -0.49 & $* * *$ \\
$\Delta l=1$ & -0.38 & $* * *$ \\
$L m=0$ & -0.35 & $* * *$ \\
$L m=1$ & 0.66 & $* * *$ \\
$O D e p=0$ & 0.21 & $* * *$ \\
$C V=0.2$ & -0.65 & $* * *$ \\
$K$ & 4.93 & $* * *$ \\
$L s=0$ & -0.40 & $* * *$ \\
$L s=1$ & 0.64 & $* * *$ \\
$\Delta t=1: \Delta l=1$ & 0.14 & $* * *$ \\
$\Delta t=1: O D e p=0$ & -0.22 & $* * *$ \\
$\Delta l=1: O D e p=0$ & -0.13 & $* * *$ \\
$\Delta l=1: L s=0$ & -0.23 & $* * *$ \\
$\Delta l=1: L s=1$ & 0.35 & $* * *$ \\
$L m=0: L s=0$ & -0.17 & $* *$ \\
$L m=1: L s=0$ & 0.42 & $* * *$ \\
$L m=0: L s=1$ & 0.33 & $* * *$ \\
$L m=1: L s=1$ & -0.68 & $* * *$ \\
$O D e p=0: C V=0.2$ & 0.13 & $* * *$ \\
$L m=1: C V=0.2$ & -0.27 & $* * *$ \\
$\Delta t=1: L s=0$ & -0.15 & $* *$ \\
$\Delta t=1: L s=1$ & 0.18 & $* *$ \\
& &
\end{tabular}

434 
435 Table 5: Information on the nodes of the multivariate regressions trees (Fig. 2). The best fits are 436 the ones with the lowest $L K$ and the highest $L b i c$

\begin{tabular}{|c|c|c|c|c|}
\hline Node number & $\begin{array}{l}\text { Number of } \\
\text { adjustements } \\
\text { in the node }\end{array}$ & $\begin{array}{l}\text { Mean Lbic (centred } \\
\text { and reduced) }\end{array}$ & $\begin{array}{l}\text { Mean } L K \\
\text { (centred and } \\
\text { reduced) }\end{array}$ & Deviance \\
\hline 1 & 648 & 0.00 & 0.00 & 1294 \\
\hline 2 & 324 & 0.26 & -0.83 & 363 \\
\hline 3 & 324 & -0.26 & 0.83 & 72 \\
\hline 4 & 108 & -0.17 & -1.46 & 195 \\
\hline 5 & 216 & 0.48 & -0.51 & 445 \\
\hline 6 & 108 & -0.51 & 0.10 & 97 \\
\hline 7 & 216 & -0.14 & 1.19 & 251 \\
\hline 8 & 144 & -0.48 & 1.20 & 134 \\
\hline 9 & 72 & 0.53 & 1.16 & 26 \\
\hline 10 & 96 & -0.96 & 1.23 & 41 \\
\hline 11 & 48 & 0.49 & 1.15 & 69 \\
\hline
\end{tabular}


439 Fig. 1: box-and-whisker plots of the estimated growth rate for the different assumptions of the 440 length-structured population model. Each row of plots corresponds to an assumption of the length441 structured population model while each column corresponds to a growth rate value used to generate 442 individual growth trajectories. In each plot, the different modalities of the considered assumption 443 are plotted in abscissa, the estimated growth rate being plotted on the Y-axis. The horizontal lines 444 represent the assumed growth rate for the simulated data.

445 Fig. 2: Multivariate regressions trees of the fits of the length-structured population growth model 446 on simulated frequencies when. $\mathrm{n}$ indicates the number of adjustements in the leaves. The 447 parameters are defined in Table 1. The grey numbers indicate the number of the nodes, more 448 information on each node can be found in Table 5 

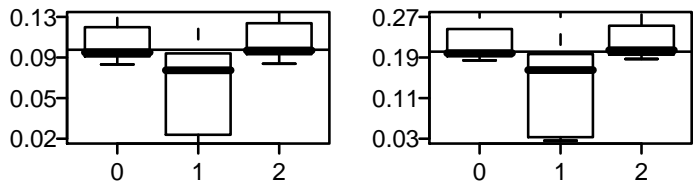

$\Delta t$
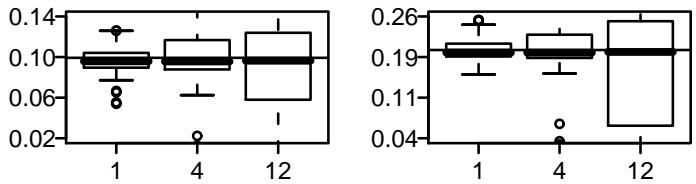

$\Delta I$
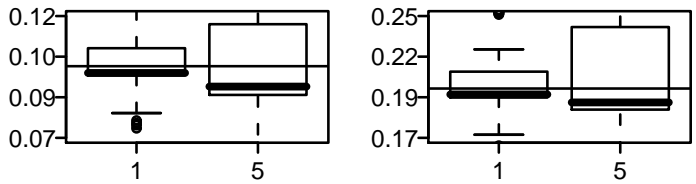

ODep
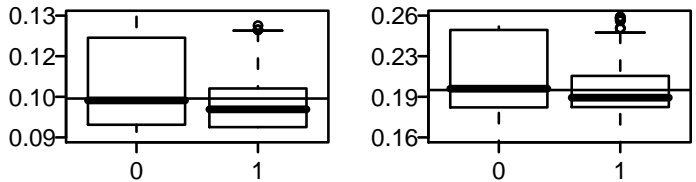

$K=0.1$

$K=0.2$ 


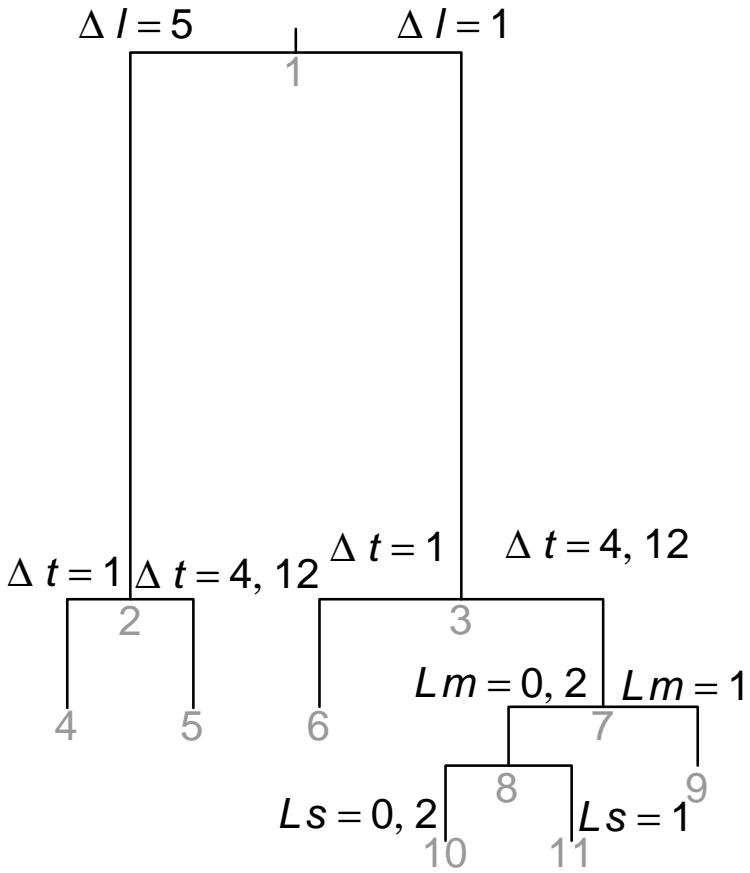

